\title{
Refresher: Akupunktur bei Kniegelenksarthrose
}

Die Therapie der Kniegelenksarthrose mit Akupunktur kann mit allen Krankenkassen abgerechnet werden, daher ist es eine der häufigen Indikationen der Akupunktur.

Arthrose ist eine westliche Diagnose, die impliziert, dass es zu einer dauerhaften Schädigung der Gelenkflächen gekommen ist und eine Heilung auf konservativem Weg nicht zu erzielen ist. Trotzdem sollte es in der Akupunkturbehandlung unser Ziel sein, eine über den Behandlungszeitraum reichende Besserung der Beweglichkeit und Reduzierung von Schmerzen zu erreichen. Dazu benötigen wir eine chinesische Syndromdiagnose. Bei chronischen Erkrankungen, bei denen die innere Ebene (Knochen) betroffen ist, müssen wir festlegen, welche Wandlungsphase/n oder Organ/e in welcher energetischen Form betroffen ist/sind und daraus die Therapie der Syndromstörung ableiten. Schon in der Anamnese können wir dazu die entscheidenden Hinweise vom Patienten erhalten.

\section{Anamnese}

Erstes Ziel sollte die Zuordnung der Beschwerden zur betroffenen Leitbahn ggf. sogar zum Punctum maximum (P.m.) des Schmerzes sein. Am eigenen Knie oder besser an einem Akupunkturmodell kann uns der Patient dies zeigen. Durch die vorhandenen Punkte und Leitbahnen erfolgt eine klare Festlegung.

\section{Diagnose}

Viele Aspekte helfen uns beim Stellen der Syndromdiagnose. Zuerst sollte die Überlegung erfolgen, welches Gewebe betroffen ist.

Knochen wird von der Niere genährt, eine chronische Störung lässt eine LeereStörung erwarten. Wir teilen das Ausmaß der Leere in Qi-, Yang- und Yin-Leere ein. Vereinfacht kann man sagen, die Qi-Ebene zeigt leichte Symptome, ohne wesentliche chronische Zeichen und ohne Veränderung von Zunge und Puls. Die Yang-Ebene zeigt Zeichen des Energieverlusts, Organfehlfunktionen und Kältezeichen. Bei der Kniearthrose oft Kälte der Knie, der LWSRegion, heller klarer Urin, die Beschwerden profitieren von Wärmebehandlung. Die Zunge ist blass, geschwollen oder normal groß, der Puls ist langsam und tief. Bei der Yin-Ebene gehen die verstärkten Zeichen der Schwäche mit Zeichen der nur äußeren Wärme einher. Charakteristisch ist die „Hitze der fünf Herzen“: Handflächen, Fußsohlen und Dekolleté. Die Zunge ist rot, gefurcht, ohne Belag.

Die Milz nährt das Bindegewebe und die Muskulatur, sie wandelt Feuchtigkeit um und macht diese ausscheidbar. Kniebeschwerden mit einem Erguss, Mitbeteiligung des Bandapparats oder der Menisken lassen zusätzlich an eine chronische Milzstörung denken. Ist die Milz geschwächt, gibt es immer auch Beschwerden des Verdauungstrakts, wie Meteorismus, wechselnde Stühle, eher dünn. Adipositas, teigige Hautstruktur, Varizen sind weitere Zeichen der Milz-Leere. Bei der Milz-Yang-Störung imponiert wieder die Kälte, die Zunge ist blass, geschwollen ggf. mit Zahneindrücken, Puls schlüpfrig. Erste Konsequenz der Diagnose ist es, auch die Ernährungsstörung anzugehen und über eine dauerhafte Ernährungsänderung eine weitere Schwächung der Milzenergie zu vermeiden.

Eher selten bei der Arthrose kann eine Leber-Stagnation zusätzlich auftreten. Kennzeichnend ist eine gut trainierte, aber deutlich verspannte Muskulatur bei oft jüngeren Patienten. Sportler mit immer gleichen Trainingsbelastungen sind hier betroffen. Hypertonie, Kopfschmerz, schmerzhafte Regelstörungen sind hinweisend. Oft kann man die Emotion Wut oder Ärger eruieren. Die Zunge zeigt rote Ränder, der Puls ist voll und schnell.

\section{Untersuchung}

Bitte achten Sie lokal auf Wärme oder Kältezeichen, Erguss, schmerzhafte Bewegungseinschränkung. Die lokalen Befunde sollten sich im Verlauf der Behandlung normalisieren. Vor der Behandlung sollte auch das Betrachten der Zunge und Tasten des Pulses erfolgen. Für Patienten ist diese chinesische Form der Untersuchung ein wesentliches Qualitätskriterium der Akupunkturbehandlung. Hier lassen sich auch weitere Untersuchungen auf die o.g. Zeichen der Niere, Milz und Lebersymptome durchführen.

\section{Behandlung}

Bitte vereinbaren Sie mit Ihrem Patienten ein gemeinsames realistisches Behandlungsziel für seine Kniearthrose. $\mathrm{Zu}$ hohe Erwartungen des Patienten führen zu Unzufriedenheit mit Ihnen und der Akupunkturbehandlung.

\section{Lagerung}

Für eine Kniegelenksbehandlung eignet sich die Rückenlage des Patienten mit erhöhtem Oberkörper und Knierolle am besten. Sie benötigen entspannte Beinmuskeln, um schmerzarm nadeln zu können.

\section{Punktauswahl}

Ich plane meine Punkte nach den folgenden Kategorien, behandele dann aber von Kopf zum Fuß mit möglichst wenig Umlagerung des Patienten und die von mir abgewandte Seite zuerst. Punkte des größten Schmerzes sollten erst nach einer ableitenden Fernpunkttherapie gestochen werden oder die Gegenseite genutzt werden.

\section{Lokalpunkte}

Sie liegen im Bereich der als schmerzhaft angegeben Knieregion ( Abb. 1). Ihre Behandlungsindikation ergibt sich durch Druckschmerz beim Untersuchen des Punktes. Sie werden bei einseitigen Beschwerden auch nur einseitig behandelt, ihre Zahl nimmt über die Behandlungsdauer tendenziell ab. Mögliche Lokalpunkte: Mi 9, Mi 10, Ma 36, Ma 35, Ex-BF 2, ExBF 4, Le 8, Gb 34, Bl 40 


\section{Achsenfernpunkte}

Aus der Bestimmung des P.m. des Schmerzes und ggf. seiner Ausstrahlung ergibt sich die betroffene Leitbahn. Auf dieser liegt ein Fernpunkt, der 2. auf der gekoppelten Leitbahn (Arm/Bein). Meist ist beim Knie die Magenleitbahn betroffen, die Dickdarmleitbahn ist der Achsenpartner. Alle Achsenfernpunkte liegen im Bereich Ellbogen- oder Kniegelenksfalte bis Handoder Fußbereich. Je proximaler der Beschwerdebereich, desto distaler liegt der Fernpunkt. Kopfbereich an Hand und Fuß, HWS bis Brustwarzenhöhe Unterarm-Unterschenkelbereich. Rest des Körpers: Brustwarzen bis Knie und Ellbogenspalt nahe am Gelenkspalt. Beim Achsenfernpunkt für das Knie sind wir im frontalen Umlauf. Für laterale Kniebeschwerden Ma 36 und Di 10, für mediale Kniebeschwerden Mi 9 und Lu 5. Diese Punkte werden immer beidseits behandelt und bleiben innerhalb der Behandlungsindikation gleich.

\section{Symptomatische Punkte}

Je nach Symptom werden diese Punkte beidseitig behandelt. Je nach Auftreten der Symptome sind sie von Sitzung zu Sitzung verschieden.

- Schmerzpunkte: Di 4, Ma 44 (ventral), Dü 3 (dorsal)

- Wind-Punkte: Gb 20, Gb 31, Bl 40

- Meisterpunkt der Knochen: Bl 11

- Treffpunkt aller Yang-Leitbahnen: LG 14

- Meisterpunkt der Muskeln und Sehnen: Gb 34

- Sedierende Punkte: He 7, Bl 62

- Öffnungspunkte der außerordentlichen Leitbahnen: Konzeptionsgefäß (frontal): Lu 7; Lenkergefäß (dorsal): Dü 3

- OP nach Projektionsareal des Schmerzes, OP 55 Shen Men, OP 26a Thalamus

\section{Punkte zur Behandlung einer Disharmonie}

Auch dies sind Fernpunkte. Sie finden nur bei chronischen Störungen auf der Organebene Anwendung. Es sind Punkte aus der Gruppe der Steuerungspunkte mit einer tonisierenden Wirkung auf Organe oder Funktionen, z.B. Shu- oder Yuan-Punkte, die meist in Kombination behandelt werden. Diese Punkte bleiben über einen Behandlungszyklus gleich.
Punkte bei Nieren-Yang-Leere: Ni 3 (Yuan), Bl 23 (Shu), LG 4 (Ming Men), Wärmetherapie auf Ni 3, LG 4, Bl 23, oder vom Patienten selbst zuhause KG 8, Ni 3, Ni 7 (Tonisierungspunkt Ni)

Punkte bei Milz-Qi- oder Yang-Leere: Mi 3 (Yuan), Bl 20 (Shu), Mi 6 (GruppenLou, Yin am Bein), Mi 4 (ÖP Chong Mai), bei gynäkologischen Symptomen zusätzlich (Menopause), Le 13 (MP Zang-Organe), KG 12 (MP Fu-Organe)

Punkte bei Leber-Qi-Stagnation oder aufsteigendem Leber-Qi: Di 4, Le 3 (Vier Tore), LG 20, bei Leber-Wind Gb 34, Le 14 (Mu der Leber), Pe 6 befreit von Thoraxbeklemmung

Zusätzlich können folgende Techniken angewandt werden:

- Elektrostimulation an Nadeln

- Wärmetherapie an Myogelosen oder zur Behandlung der Disharmonie

- Akutaping am Knie, ggf. Ergusstape

- OP 40 LWS, OP ISG-Gelenk, OP 49 Kniegelenk, OP 26a Thalamus, OP 55 Shen Men, psychotrope Punkte (z.B. Antiaggressionspunkt, Vegetativum I)

Die Punktauswahl muss pro Behandlung dokumentiert werden, die Aufklärung zur Behandlung ebenfalls. Einige KVen haben dazu eigene Qualitätsbögen vorgeschrieben, diese sind dann bei Abrechnung im EBM zu führen.

Die Liegezeit mit Nadeln beträgt meist 20 min, kann aber je nach Patientenwunsch und Indikation länger und kürzer ausfallen. Kurze Behandlungszeiten mit sedierender Nadeltechnik oder Reizstrom und Schröpfen werden bei akuten Störungen angewandt. Sanftes Nadeln überwiegend tonisierender Punkte bei langer Liegezeit ist die häufigere Anwendungsform bei Patienten mit chronischem Krankheitsverlauf. Zusätzlich kann lokale Wärmetherapie, Ernährungstherapie und ggf. chinesische Phytotherapie ergänzt werden.

Nach 4-6 Behandlungen sollte sich ein erster Erfolg bei chronischen Knieschmerzen eingestellt haben, sonst muss das Therapiekonzept überdacht werden. Ist nach 10 Behandlungen ein ausreichender Therapieerfolg erreicht, sollte die Therapie erst nach einer Pause bei erneuter Beschwerdeverstärkung bis zur 15. Behand-

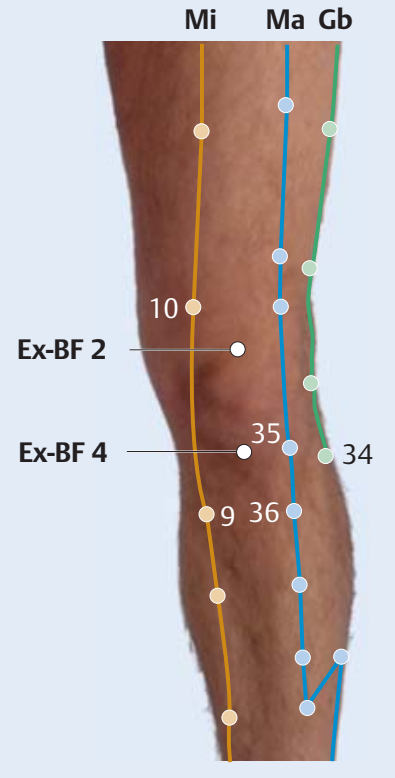

Abb. 1 Lokalpunkte am Knie. (c) [1].

lung fortgesetzt werden. Diese Empfehlung richtet sich leider mehr nach $\mathrm{Ab}$ rechnungsregeln als nach medizinischen Patientenbedürfnissen. Die EBM-Regeln lassen sich aber nicht ignorieren. Die erreichten Ziele und das weitere Prozedere sollten in einem Abschlussgespräch mit dem Patienten besprochen werden.

\section{Literatur}

[1] Velling P, Peuker ET, Steveling A, Hecker H-U. Checkliste Akupunktur. 2. Aufl. Stuttgart: Hippokrates; 2009

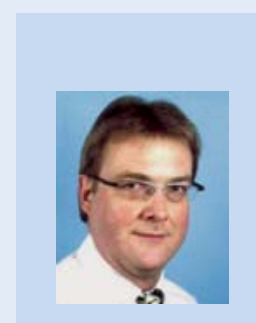

Dr. med. Peter Velling

MVZ der Ev. Lungenklinik Berlin

Bergmannstraße 5

10961 Berlin-Kreuzberg

peter.velling@elk-berlin.de

Ausbildung zum Internisten, Allergologie, Akupunktur an der Med. Univ. Poliklinik in Bonn. Von 1996 bis 2008 als hausärztlicher Internist niedergelassen in Bonn. Seit 2009 Koordinator des Ambulanzbereichs der Ev. Lungenklinik Berlin. 